\title{
The differences in energy system demands of competitive male tennis play between fast and slow courts
}

\author{
Anna Skorodumova ${ }^{a}$ (iD $\&$ Igor Baranov ${ }^{a}$ \\ ${ }^{a}$ Federal Science Center for Physical Culture and Sport, Moscow, Russia.
}

\section{ABSTRACT}

This study investigated the energy system demand differences between matches played on fast courts and on slow courts of high-performance male players. Matches of Grand Slam matches played by Russian players were analysed. External indicators as such in-point playing time as a proportion of total match time were used to conduct the analysis. Data analysis found significant differences in all measured external indicators such as average match playing time, number of points and in-point time. Results also found a significant difference between match motor density (the percentage of total match time that made up effective playing time) for the two surface types, with the slow surface (clay) match motor density being significantly higher than that of the fast surface (grass and hard court), $14.5 \%$ and $12.9 \%$, respectively. Subsequent analysis of point length reveals clay courts that slow courts had a significantly higher percentage of points that lasted more than 10 seconds whilst fast courts has a higher percentage of points under 10 seconds in length. It can be concluded that due to the length of the points, tennis primarily employs the ATP-PCr energy system for energy use, dipping into the anaerobic lactic acid energy pathways more on clay court matches. Whilst the aerobic pathways are not employed heavily in-point, they are still essential, possibly even more on clay court due to greater taxation on the anaerobic lactic acid pathway, as they provide the basis for ATP resynthesis between points. This signals toward endurance as a major component of fitness in tennis.

\author{
Key words: energy systems, \\ fast and slow courts, \\ metabolism, tactics. \\ Received: 20 August 2019 \\ Accepted: 07 October 2019 \\ Corresponding author: Anna \\ Skorodumova, Federal Science \\ Center for Physical Culture and \\ Sport, Moscow, Russia. Email: \\ apskorodumova@mail.ru
}

\section{RESULTS}

\section{INTRODUCTION}

Staff at the FSBI Federal Science Centre for Physical Culture and Sport conducted a study of the external indicators of energy system demands of male tennis players during competitive matches played on slow surfaces (clay courts) and fast surfaces (hard and grass courts). Grand Slam tournament matches, including the finals and semi-finals as well, in which Russian male tennis players participated, were researched and analysed.
The average external indicators of energy demands obtained from the study of the matches are presented in table 1. 
Table 1. Average external indicators of energy system demand of male competitive play on fast and slow courts

\begin{tabular}{|l|l|l|l|}
\hline Indicator & Surface Type & Difference \\
s
\end{tabular}

the time, players prepare for the serve or return, change sides of the court, or rest, etc.

During actual in-point play time, 193-206 points are played depending on court surface type on average, and the profile of the duration of the points played on each court type is also different.

The percentage profile of whether the points were less than or more than 10 s in length for each surface type are presented in table 2. Research from recent years shows that after 6-10s of close to maximum intensity work, stocks of creatine phosphate diminish to the point that the creatine phosphate reaction stops being the main mechanism of ATP resynthesis (Cheremisinov ,2016; Jansen et al., 2016), and the longer the work is, the bigger the role of lactic acid anaerobic sources in power supply.

Table 2. Table 2. Percentage profile of points being either less or more than 10 seconds in length in matches of male tennis players on fast and slow courts

\begin{tabular}{|l|l|l|}
\hline \multirow{2}{*}{ Surface type } & \multicolumn{2}{|c|}{ Different duration points quantity, \% } \\
\cline { 2 - 3 } & $\leq 10 \mathrm{~s}$ & $>10 \mathrm{~s}$ \\
\hline Fast & $86,82 \pm 4,36$ & $13,18 \pm 4,36$ \\
Slow & $83,20 \pm 5,31$ & $16,80 \pm 5,31$ \\
\hline Differences & Significant & Significant \\
\hline
\end{tabular}

\section{DISCUSSION}

Thus, in a game on fast-paced courts, tennis players play larger percentages of points less than 10s in length, than on slow courts, and on slow courts, the percentage of the points played lasting more than $10 \mathrm{~s}$ was more than on fast courts. It is important to note that the differences between the percentages of points played that are under 10s and over 10s on fast and slow courts are statistically significant and they need to be considered when drawing up plans of training.
The average values of match motor density (the ratio of actual in-point play time to the total match time, expressed as a percentage) demonstrate that for only $14.5 \%$ and $12.9 \%$ of match time on average, depending on court surface type, are tennis players actually engaged within a point. For the rest of 


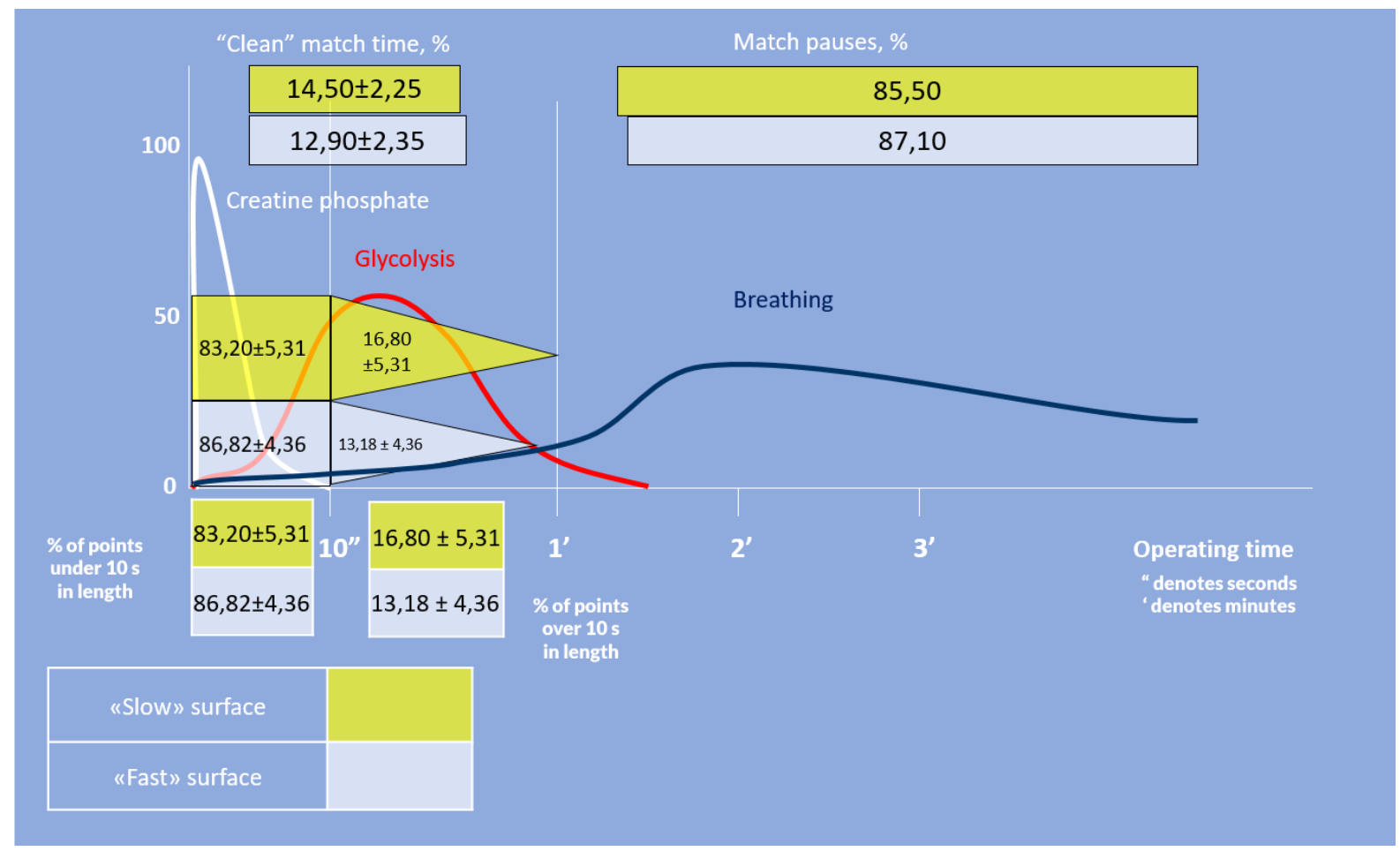

Figure 1. The use of different energy systems for ATP re-synthesis during tennis matches of men on different surfaces, split by length of point (\% of time)

Therefore, of $14.5 \%$ of match time on a slow and $12.9 \%$ on a fast pace court surface (clean match time or in-point effective playing time), 83.2-86.8\% depending on court surface type could be seen to be met primarily with the anaerobic lactic acid free energy system - the ATP-PCr pathway, whilst 16.8$13.18 \%$ of effective play time energy needs are provided by lactic acid anaerobic systems (Cheremisinov,2016; Jansen et al., 2016). 85.5-87.1\% of match time (out-of-point time), depending on the type of court surface, is provided with aerobic abilities and, of course, readiness of the athlete to play the following point will depend on their power and efficiency.

Schonborn (1987) considers that the different energy systems are involved in tennis in the ratios of: anaerobic lactate free (ATP-PCr) - 70\%, anaerobic lactic acid - $20 \%$, and aerobic 10 \%). It would be possible to agree with this statement if a match was only one point in length.

However, in our opinion, big requirements are imposed on aerobic abilities in a match due to the fact that for more than $80 \%$ of match time, they provide the player with ATP resynthesis after each point. The more intense and the longer the point, the higher the rate of aerobic transformations. The leading contributor in rate of aerobic process speed is the ATP/ADP ratio - from the beginning of intense muscular activity and with further increases in intensity, concentration of ATP decreases, and ADP increases.
It is necessary to pay tribute to Roger Federer and experts working with him. Training is conducted in such a way that in a match, on average $73 \%$ of all points are 4 shots or less. Points $5-8$ shots in length occur $19 \%$ of the time. Only in $8 \%$ of cases is a point 9 shots or longer (Perov, 2019). It means that $92 \%$ of all points are conducted at the expense of the lactic free anaerobic abilities (ATP-PCr) and only in $8 \%$ of cases, the lactic anaerobic energy system begins to become more active. For comparison, the author provides average values of number of points at different lengths for Novak Djokovic - up to 4 shots in $55 \%$ of cases, $5-8$ shots in $26 \%$ of cases and 9 or more shots in $19 \%$ of cases, which testifies to a larger possibility of lactic acid build-up, so and bigger requirements to the aerobic productivity of the athlete.

It should be noted that a game on a slow surface shows to hav'e even bigger requirements on the aerobic abilities of tennis players, than on fast, since a higher proportion of time is s jent actually in-point play. Even though points may be longer on slow-paced courts, with bigger expenditure of creatine phosphate stores, according to rules of competition, a breaks in play are the same length. During this pause it is necessary to resynthesise creatine phosphate. The greatest coritributor to ATP resynthesis is played by aerobic oxidation processes in this case.

Movements during a point can be characterised as high in reaction speed, acceleration, single movement speed, movement frequency (Godik, 2006; Zatsiorsky, 1966); the movements are high-speed and powerful requiring a full array of cleveloped coordination abilities (balance, accuracy, 
differentiation of muscular efforts, etc.). But tennis players do not play just one point but on average more than 190 points during the match, and this means that the emphasis on the importance of qualities changes. High-speed, power and coordination abilities should be maintained at a high level for long periods of time.

Endurance is the ability to perform work without a change in parameters (in tennis - without reducing intensity, accuracy, variability of actions, speed and explosiveness, etc.) despite the arising exhaustion (Matveev, 1977; Farfel, 1949; Mikhaylov, 1967; Platonov, 2014) and it begins to prevail as the most necessary component of fitness. It is also necessary for the tennis player once the match reaches about 2 hours in duration.

Another aspect that signals to endurance as a major component of fitness is tennis is that the qualities which are necessary for a single point have to be met throughout the entire duration of the competitive activity, which means that high-speed, power, coordination and other abilities have to be maintained not just for a short period of time but long ones.

The conducted factorial analysis of physical and functional fitness of high-performance tennis players and the calculated correlation coefficients between indicators of physical and functional fitness and results of competitive activity confirm the conclusions drawn.

\section{REFERENCES}

Cheremisinov, V.N. (2016), Power ensuring of intense muscular activity, TVT Division: Moscow, Russia

Farfel, V.S. (ed.) (1949), Endurance as physiological concept "Research on Endurance Physiology", Physical culture and sport, Moscow, Lvov, USSR, 270

Godik, M.A. (2006), Physical training of football players, Olympia Press: Moscow, Russia

Jansen, P. (2016), HR, lactate and trainings on endurance, Tuloma

Matveev, L.P. (1977), Bases of sports training, Physical culture and sport: Moscow, USSR

Mikhaylov, V.V. (1967), Problems of switching in sport, TIPFK, No. 2, Moscow, USSR, pp. 40-45.

Perov, G. (2019), "Advantage of the first shots", Tennis, No. 6., Moscow, Russia, pp. 36-37.

Platonov, V.N. (2014), Periodization of sports training. General theory and its practical application, Olympic literature: Kiev, Ukraine, 624 p.

Schonborn, R. (1987), Energy systems in tennis, International Tennis Federation, ITF Ltd. Bank Lane, Roehampton, London, UK

Zatsiorsky, V.M. (1966), Physical qualities of the athlete, PIS: Moscow, USSR 